Z. klin. Chem. u. klin. Biochem.

9. Jg., S. 478-485, November 1971

\title{
Ein Verfahren zur Bestimmung der 3-Methoxy-4-hydroxy-phenylessigsäure (Homovanillinsäure) im Harn durch in situ Remissionsmessung nach dünnschichtchromatographischer Trennung
}

\author{
Von E. Knoli, H. Wisser und D. Stamm \\ Aus dem Max-Planck-Institut für Psycbiatrie, Mïnchen
}

(Eingegangen am 6. Mai 1971)

Herrn Professor Dr. G. Pexers zum 65. Geburtstag

\begin{abstract}
Es wird ein Verfahren zur Bestimmung der 3-Methoxy-4-hydroxy-phenylessigsäure (Homovanillinsäure) im Harn beschrieben, das - nach Extraktion mit Essigsäureäthylester und dünnschichtchromatographischer Trennung der Phenolcarbonsäuren - auf der in situ Remissionsmessung bei $280 \mathrm{~nm}$ beruht. Die Zuverlässigkeitskriterien der Methode wurden ermittelt. An einem Kollektiv von 45 Frauen und 49 Männern wurde die 24-Stdn.-Ausscheidung der Homovanillinsäure ermittelt. Die Prüfung auf Alters- und Geschlechtsabhängigkeit ergab bei den Frauen eine signifikant geringere Homovanillinsäureausscheidung. Die Methode wurde angewendet bei der Analyse von 3-Stdn.-Urinproben. Der diagnostische Wert der Homovanillinsäurebestimmung wird diskutiert.
\end{abstract}

\section{A method for the determination of 3-methoxy-4-bydroxy-phenylacetic acid (bomovanillic acid) in urine by the in situ remission measurement after thin layer cbromatograpbic separation}

A method is described for the determination of 3-methoxy-4-hydroxy-phenylacetic acid (homovanillic acid), which is based on the in situ remission measurement at $280 \mathrm{~nm}$, following extraction with ethyl acetate and thin layer chromatogtaphic separation of the phenylcarboxylic acids. Criteria for the reliability of the method are reported. The $24 \mathrm{~h}$ excretion of homovanillic acid was determined on a collective of 45 women and 49 men. The test for age- and sex-dependency showed that the excretion of homovanillic acid is significantly lower in women. The method was used for the analysis of $3 \mathrm{~h}$ urine samples. The diagnostic value of the homovanillic acid determination is discussed.

Die 3-Methoxy-4-hydroxy-phenylessigsäure (Homovanillinsäure) wurde 1956 von ARMSTRONg und Mitarbeitern als ein normales Ausscheidungsprodukt im menschlichen Urin nachgewiesen (1). In Analogie zum Abbau des Adrenalins und Noradrenalins zur 3Methoxy-4-hydroxy-mandelsäure (Vanillinmandelsäure) entsteht sie als Hauptabbauprodukt des Dopamins durch Einwirkung der Enzyme Catechol-O-methyltransferase (EC 2.1.1.6) und Monoaminoxydase (EC 1.4.3.4) $(2,3)$.

Infolge der Viélzahl an chemisch sehr ähnlichen Stoffwechselprodukten der Katecholamine ist eine direkte Bestimmung der Homovanillinsäure im Urin oder auch in einem Urinextrakt nicht möglich, da es keine spezifische Nachweisreaktion für sie gibt. Der eigentlichen Nachweisreaktion müssen deshalb noch Trennoperationen vorausgehen. Folgende Methoden wurden bisher angewandt: Ein- und zweidimensionale Papierchromatographie $(1,2,4,5)$, die Dünnschichtchromatographie $(6,7,8)$, die Hochspannungspapierelektrophorese (9) und die Gaschromatographie (10-13). In alkalischem Milieu läßt sich die Homovanillinsäure durch Oxydation mit Fe-(III)-Salzen in einen Fluorophor überführen. Sharman (14) sowie ANDÉN und Mitarbeiter (15) wandten diese Reaktion zu einer fluorometrischen Homovanillinsäure-Bestimmung in Gewebehomogenaten an. Nach dem gleichen Prinzip arbeitete SATO (16), der nach Ionenaustauschchromatographie und Lösungsmittelextraktion die Homovanil- linsäure im Urin bestimmte. Von RUTHVEN und SANDLER stammen zwei Methoden, die darauf beruhen, daß die Homovanillinsäure durch teilweisen Abbau in leichter bestimmbare Substanzen überführt wird. In der ersten umständlicheren Methode (17) wird im Autoklaven mit $\mathrm{HBr} /$ Eisessig demethyliert und die entstandene Dihydroxyphenylessigsäure nach Adsorption an Aluminiumoxid und Elution photometrisch bestimmt. Bei der zweiten einfacheren Methode (18) wird die Homovanillinsäure ebenfalls im Autoklaven mit Kupfer und Silikagel in Vanillin überführt und dieses photometrisch gemessen. In Urinextrakten ist die Umwandlung in Vanillin jedoch nicht quantitativ.

Die Nachteile der bisherigen Bestimmungsmethoden für Homovanillinsäure bestehen in dem zu großen Arbeitsaufwand oder der mangelnden Spezifität. Ziel der vorliegenden Arbeit war, ein möglichst einfaches Verfahren von ausreichender Präzision und Richtigkeit zu entwickeln, das sowohl zur Analyse von 24-StundenUrinproben, wie auch von 3-Stunden-Urinproben bei der Untersuchung circadianer Rhythmen eingesetzt werden kann.

$\mathrm{Da}$ die Ausscheidung der Homovanillinsäure im 24 Stunden-Urin im Milligramm-Bereich liegt, ist es nicht erforderlich, ein fluorometrisches Verfahren $\mathrm{zu}$ verwenden. Geeignet schien uns die dünnschichtchromatographische Trennung der Phenolcarbonsäuren nach Lösungsmittelextraktion des Urins. Statt des bisher üblichen Besprühens mit einem Farbreagenz und an- 
schließender Elution $(6,7)$, wurde die in situ Remissionsmessung im Absorptionsmaximum der Homovanillinsäure bei $280 \mathrm{~nm}$ durchgeführt.

Bei dieser Methode wird nur die freie Homovanillinsäure bestimmt. Eine Hydrolyse des Urins vor der Lösungsmittelextraktion ist nicht notwendig, da der konjugierte Teil nur 4-12\% des freien Anteils beträgt (19). PETRÁšEK und Mitarbeiter ${ }^{\circ}$ (20) geben an, $\mathrm{da} \beta$ dieser Anteil etwa $1 / 3$ beträgt, $\mathrm{da} \beta$ aber die Ausscheidungskurve der konjugierten Homovanillinsäure derjenigen der freien Homovanillinsäure parallel verlaufe. SHaw und Mitarbeiter (2) finden bei der Analyse eines 8-Stunden-Urins $1,2 \mathrm{mg}$ freie Homovanillinsäure und $0,6 \mathrm{mg}$ konjugierte Homovanillinsäure.

\section{Methodik}

Der Urin wird bei $\mathrm{pH}$ 1-2 mit Essigsäureäthylester ausgeschüttelt. Hierbei gehen die Phenolcarbonsäuren in die organische Phase über. Nach Eindampfen des Extraktionsmittels und Aufnahme in einem kleinen Volumen Athanol/Wasser wird das Substanzgemisch dünnschichtchromatographisch getrennt. Die Auftragung auf die Platte erfolgt bandförmig mit dem ,Linomat" nach Wässle und SandhofF (21). Die Auswertung der Platten erfolgt durch in situ Remissionsmessung bei $280 \mathrm{~nm}$ ohne vorhergehendes Anfärben der aufgetrennten Substanzen.

Apparate und Glasgeräte

1. $50 \mathrm{ml} \mathrm{Zentrifugengläser,} \mathrm{mit} \mathrm{NS} 29,11 \mathrm{~cm}$ hoch, die unten spitz ausgezogen sind (22).

2. Quickfit-Austeilautomat für organische Lösungsmittel ${ }^{1}$ ).

3. Schüttelmaschine (22).

4. $20 \mathrm{ml}$ Recordspritze mit abgestumpfter Nadel.

5. Wasserbad $75^{\circ}$.

6. Rotationsverdampfer, Rotavapor ${ }^{2}$ ).

7. $2 \mathrm{ml}$ Spritze mit $30 \mathrm{~cm}$ langer Kanüle aus V2A-Stahl.

8. Meßpipetten, $0,5 \mathrm{ml}, 1 / 200-$ Teilung $^{3}$ ).

9. Camag-Beschichtungsautomat ${ }^{4}$ ).

10. Camag-Linomat nach WäSSLE und SANDHOFF ${ }^{4}$ ).

11. Chromatogramm-Spektralphotometer ${ }^{5}$ ).

12. Kompensationsschreiber Servogor, Modell Re 5116).

\section{Reagenzien}

Wenn nicht besonders vermerkt, werden p. a. Reagenzien der Firma Merck, Darmstadt, benutzt.

Essigsäureäthylester p. a.

Atthanol p. a.

Essigsäure, min. 96proz.

Benzol p. a.

Salzsäure, 18proz.

Kieselgel $\mathrm{H}$ nach StaHL, füṛ die Dünnschichtchromatographie Homovanillinsäure, puriss? ${ }^{7}$ ).

Homovanillinsäure-Standardlösungen:

$2 \mathrm{mg}, 4 \mathrm{mg}$ und $6 \mathrm{mg}$ Homovanillinsäure/1 demin. Wasser.

Hiervon werden jeweils $10 \mathrm{ml}$ zur Analyse eingesetzt. Diese Lösungen sind, lichtgeschützt aufbewahrt, bei Raumtemperatur mindestens 4 Wochen stabil.

1) Quickfit Laborglas GmbH, Wiesbaden-Schierstein.

2) Firma Büchi, 9230 Flawil, Schweiz.

3) Firma Link, Wertheim.

4) Firma Camag, Muttenz/Schweiz.

5) Firma Carl Zeiss, Oberkochen/Württ.

6) Firma Metrawatt AG., Nürnberg.

7) Fluka AG., Buchs/Schweiz.

\section{Arbeitsweise}

\section{Bescbichten und Aktivieren der Platten}

$35 \mathrm{~g}$ Kieselgel $\mathrm{H}$ (für 10 Platten) werden in $80 \mathrm{ml}$ Wasser suspendiert und 5 Min. kräftig geschüttelt. Dann werden die Platten in $250 \mu \mathrm{m}$ Dicke mit dem Camag-Beschichtungs-Automaten beschichtet. Nach Lufttrocknen werden die Platten 2 Stdn. bei $120^{\circ}$ aktiviert. Um Verschmutzung durch die Laboratoriumsluft zu vermeiden, werden die Platten in einem luftdicht verschließbaren Plattenaufbewahrungsschrank über Calciumchlorid aufbewahrt. Unter diesen Bedingungen sind dic Platten mindestens 14 Tage verwendbar.

\section{Extraktion, Cbromatographie}

$10 \mathrm{ml}$ filtrierter Urin werden in ein $50 \mathrm{ml}$ Zentrifugenglas pipettiert und mit 18proz. Salzsäure auf $\mathrm{pH} 1-2$ eingestellt. Nach Zugabe von $30 \mathrm{ml}$ Essigsäureäthylester wird die Probe $10 \mathrm{Min}$. geschüttelt. Die Unterphase wird mit einer $20 \mathrm{ml}$ Recordspritze abgezogen und verworfen. Anschließend wird die Probe 1 bis 2 Min. bei 3000 U./Min. zentrifugiert. Reste der wäßr. Phase werden dann vollständig entfernt. Die organische Phase wird am Rotationsverdampfer bei $75^{\circ}$ bis zur Trockene eingedampft. Dazu wird eine "Spinne" benutzt, so daß vier Proben gleichzeitig eingeengt werden können. Der Rückstand wird in $1 \mathrm{ml}$

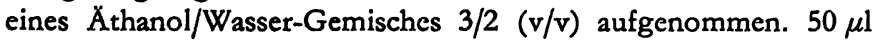
hiervon werden bandförmig in $1,4 \mathrm{~cm}$ Breite auf die beschichtete Platte aufgetragen. Dazu wird das Auftraggerät "Linomat" in Verbindung mit einer Meßpipette $0,5 \mathrm{ml}$ (1/200-Teilung, mit Schellbachstreifen) benutzt. Zum blasenfreien Füllen der Pipette wird eine $2 \mathrm{ml}$ Spritze mit einer $30 \mathrm{~cm}$ langen Kanüle verwandt. Nach dem Auftragen einer Probe werden Pipette und Düse des Auftraggerätes mit 1,5-2 ml einer 60proz. wäßr. Äthanollösung gespült. Auf eine Platte werden jeweils 3 Standards verschiedener Konzentration und 4 Urinextrakte aufgetragen. Die dünnschichtchromatographische Trennung erfolgt bei Kammersättigung durch dreimalige Chromatographie in der Oberphase eines Gemisches Benzol/Eisessig/Wasser $=2 / 2 / 1(\mathrm{v} / \mathrm{v})$. Die Laufstrecke beträgt jeweils $15 \mathrm{~cm}$. Die Dauer einer Chromatographie beträgt etwa 35 Min. Die Platte wird mit einem Föhn 10 Min. getrocknet und danach kann sofort mit dem Chromatogramm-Spektralphotometer bei $280 \mathrm{~nm}$ die Remissionsortskurve gemessen werden. Zur Registrierung dient ein Kompensationsschreiber. Zur besseren Auswertbarkeit der Peaks läuft der Papiervorschub am Schreiber mit der vierfachen Geschwindigkeit des Kreuztisches, so daß die resultierende Remissionsortskurve im Verhältnis $4: 1$ gedehnt ist.

\section{Meßbedingungen am Chromatogramm-Spektralphotometer}

Meßspalt $14 \times 0,5 \mathrm{~mm}$.

Verstärkung $6 / 1 / \mathrm{I} / \mathrm{F}$ (auf der Schalterstellung $\mathrm{F}$ ist eine Zusatzverstärkung [Faktor 6,5] eingebaut).

Kreuztischgeschwindigkeit: $30 \mathrm{~mm} / \mathrm{Min}$. (Stufe 3).

Schreiberanschluß am Anzeigegerät: $10 \mathrm{mV}$.

Papiervorschubgeschwindigkeit am Schreiber: $120 \mathrm{~mm} / \mathrm{Min}$.

Meßbereichschalter am Schreiber: $5 \mathrm{mV}$.

Schiebeschalter am Schreiber: calib.

Standards: Von den 3 wäßr. Homovanillinsäure-Standardlösungen unterschiedlicher Konzentration werden jeweils $10 \mathrm{ml}$ zur Analyse eingesetzt und mit $50 \mu \mathrm{l} 18 \mathrm{proz}$. Salzsäure versetzt. Der weitere Arbeitsgang verläuft wie bei den Urinproben.

\section{Auswertung}

Bei Remissionsmessungen ist allgemein die KubelKa-MunkFunktion die Grundlage zur quantitativen Auswertung. Die lineare Abhängigkeit der KUBELKA-MuNK-Funktion von der applizierten Substanzmenge konnte gezeigt werden $(23,24)$. Diese Art der Auswertung ist für routinemäßige Remissionsmessungen zu kompliziert. Es hat sich jedoch gezeigt, daß für die meisten Stoffe in einem bestimmten Konzentrationsbereich eine lineare Beziehung zwischen der Fläche der Remissionsortskurve und der Quadratwurzel der Substanzmenge pro Fleck besteht 
$(25,26)$. Die hicraus resultierenden Eichgeraden gehen allerdings nicht durch den Koordinatennullpunkt.

Für die Homovanillinsäure gilt diese lineare Beziehung im Bereich von $0,5-15 \mu \mathrm{g}$ Homovanillinsäure pro Fleck; größere Mengen Homovanillinsäure wurden nicht geprüft. Da sowohl die Steigung als auch der Ordinatenabschnitt der Eichgeraden von Platte zu Platte variiert, muß diese für jede Platte neu bestimmt werden; dies geschieht mit den 3 wäßr. HomovanillinsäureStandardlösungen. Die Konzentration dieser Standards ist so getvählt, daß in $50 \mu \mathrm{l}$ aufgetragerier äthanol. Lösung 1,2 und $3 \mu \mathrm{g}$ Homovanillinsäure enthalten sind. Bei der oben beschriebenen Arbeitsweise liegen im Normalfalle fast alle Urinproben in diesem Konzentrationsbereich. $\mathrm{Da}$ die Peaks eine ausreichend symmetrische Form zeigen, wird ihre Fläche nach der Formel "Höhe $\times$ Halbwertsbreite" ermittelt.

Mit Hilfe der Eichgeraden erhält man die $\mu$ g Homovanillinsäure/ $50 \mu \mathrm{l}$ äthanol. Lösung. Die Gesamtmenge Homovanillinsäure/ 24-Stunden-Urin berechnet sich nach folgender Beziehung:

$$
c=\frac{2 a . b}{1000}(m g \text { Homovanillinsäure/24-Stdn.-Urin) }
$$

$\mathrm{a}=\mu \mathrm{g}$ Homovanillinsäure $/ 50 \mu \mathrm{l}$ äthanol. Lösung.

$\mathrm{b}=$ Volumen des 24-Stunden-Urins in ml.

\section{Erläuterungen}

Beim Eindampfen des Essigsäureäthylesters soll die Wasserbadtemperatur $70^{\circ}$ nicht wesentlich unterschreiten, da sonst das Vakuum erhöht werden muß. Dadurch wird aber in den engen Zentrifugengläsern die Gefaht des "Stoßens" erhöht. Bei sehr langsamem Einengen von reinem Essigsäureäthylester zeigen sich im Chromatogramm zwei Artefakte. Diese interferieren jedoch nicht mit der Homovanillinsäure, da ihre Laufstrecken deutlich von der der Homovanillinsäure verschieden sind. Mit $1 \mathrm{ml}, 60$ proz. wäßr. Äthanollösung gelingt es nach sorgfältigem Benetzen der gesamten inneren Oberfläche des Zentrifugenglases, die Homovanillinsäure aus dem Eindampfrückstand vollständig aufzunehmen. Mit kleineren Volumina ist dies nicht mehr gewährleistet. Andererseits sind größere Volumina ungünstig, da dann in der resultierenden Lösung die Homovanillinsäure-Konzentration zu gering wird.

Das Totvolumen der Spritzpistole am Linomat beträgt etwa $80-100 \mu \mathrm{l}$. Es ist deshalb zu empfehlen, die Pipette bis zum oberen Rand mit der Äthanollösung zu füllen, dann ungefähr $300 \mu \mathrm{l}$ abzulassen, wobei der Totraum der Spritzpistole mit der aufzutragenden Lösung gefüllt und gespült wird. Nach Einstellen auf eine bestimmte Marke werden dann $50 \mu \mathrm{l}$ auf die Platte aufgetragen. Der $\mathrm{N}_{2}$-Druck lag je nach Versuchsbedingungen zwischen 0,2 und $0,3 \mathrm{~kg} / \mathrm{cm}^{2}$. Bei höherem Druck besteht die Gefahr der Zerstörung der Kieselgelschicht. Aus dem gleichen Grund sollte der Abstand zwischen Plattenoberfläche und Düse der Spritzpistole nicht zu gering sein. Bei unseren Versuchen hat sich der Abstand von $7 \mathrm{~mm}$ bewährt.

\section{Zuverlässigkeitskriterien der Methode}

\section{Präzision}

Zur Präzisionskontrolle wurden pro Versuchsserie täglich 2 Proben eines Kontrollurins mitanalysiert. Die Herstellung des Kontrollutins erfolgte aus 101 Sammelurin durch Zugabe von $100 \mathrm{ml} \mathrm{25proz}$. Salzsäure, gute Mischung und Aufteilung in $100 \mathrm{ml}$ Portionen. Die Proben wurden eingeftoren und bis zur Analyse bei $-30^{\circ}$ aufbewahrt.

Tab. 1

Ergebnis der Präzisionskontrolle mit selbst hergestellten Kontrollurinproben

\begin{tabular}{ccc}
\hline $\begin{array}{c}\text { Statistische } \\
\text { Kénngröße }\end{array}$ & $\begin{array}{c}\text { In der Serie aus } \\
\text { Doppelbestimmungen }\end{array}$ & Von Tag zu Tag \\
\hline $\bar{x}$ (mg/l Urin) & 4,52 & 4,45 \\
s (mg/l Urin) & 0,20 & $0,41 \quad$ \\
V (\%) & 4,4 & 9,3 \\
$n$ & 14 Doppelbest. & 14 Einfachbest. \\
\hline
\end{tabular}

Aus den Differenzen der Doppelbestimmungen wurde die Streuüng in der Seric berechnet, für die Strcuung von Tag zu Tag wurden die jeweils ersten Werte der Doppelbestimmungen benutzt. Das Ergebnis dieser Untersuchung wird in Tabelle 1 wiedergegeben.

\section{Richtigkeit}

\section{Wiederfindung}

$10 \mathrm{ml}$ eines Kontrollurins wurden mit $30 \mu \mathrm{g}$ und $60 \mu \mathrm{g}$ Homovanillinsäure aufgestockt (entsprechend 3 bzw. $6 \mathrm{mg}$ Homovanillinsäure/1 Urin). Die Ergebnisse sind in Tabelle 2 zusammengefaßt.

Tab. 2

Ergebnis von Wiederfindungsversuchen an Kontrollurinproben. Den beiden Aufstockzusätzen liegen Vierfachbestimmungen zugrunde. HVS $=$ Homovanillinsäure

\begin{tabular}{|c|c|c|}
\hline Aufstockzusatz & $\begin{array}{l}\mu g \text { HVS wiederge- } \\
\text { funden (Bereich) }\end{array}$ & $\begin{array}{c}\text { Mittl. Wieder- } \\
\text { findungsrate (\%) } \\
\text { und Bereich }\end{array}$ \\
\hline $\begin{array}{l}30 \mu \mathrm{g} \mathrm{HVS} / 10 \mathrm{ml} \\
60 \mu \mathrm{g} \mathrm{HVS} / 10 \mathrm{ml}\end{array}$ & $\begin{array}{l}24,1-29,9 \\
47,0-57,2\end{array}$ & $\begin{array}{l}89,1(80,3-99,6) \\
84,8(78,3-95,3)\end{array}$ \\
\hline
\end{tabular}

\section{Rechromatographie in einem anderen Laufmittelsystem.}

Um zu zeigen, daß der Homovanillinsäure-Peak im Chromatogramm der Phenolcarbonsäuren ausschließlich aus Homovanillinsäure besteht, wird die Rechromatographie in einem anderen Laufmittelsÿstem benutzt.

Zunächșt werden sowohl reine Homovanillinsäure-Lösung als auch ein Urinextrakt mit dem in dieser Arbeit beschriebenen Trennsystem chromatographiert. Nach genauer Markierung der Homovanillinsäure-Peaks mit dem Chromatogramm-Spektralphotometer, Abkxatzen der betreffenden Flecken und Elution mit $0,5 \mathrm{ml} \mathrm{60proz.} \mathrm{wäßr.} \mathrm{Äthanol,} \mathrm{werden} 100 \mu \mathrm{l}$ hiervon wieder auf Kieselgel $\mathrm{H}$ aufgetragen. Als Trennsystem wird nun das von TAUTZ und Mitarbeitern (7) angegebene Laufmittelsystem verwendet. Sowohl bei der reinen Homovanillinsäure als auch bei der aus dem Urinextrakt stammenden „Homovanillinsäure" ergab sich nur ein einziger Peak mit einer Laufstrecke von $5,5 \mathrm{~cm}$. Dies läßt den Schluß zu, daß schon bei der ersten Auftrennung in dem Benzol/Eisessig/Wasser-Gemisch die. Homovanillinsäure vollständig von allen anderen Phenolcarbonsäuren abgetrennt ist.

\section{Absorptions- und Remissionsspektrum von Homovanillinsäure}

Die Richtigkeit des Verfahrens wurde außerdem durch das Messen des Absorptions- und Remissionsspektrums der Homovanillinsäure geprüft.

Abbildung 1 zeigt das Absorptionsspektrum von Homovanillinsäure in äthanol. Lösung (1a), sowie die Remissionsspektren auf Kieselgel $\mathrm{H}$ von reiner Homovanillinsäure $(1 \mathrm{~b})$ und des Homovanillinsäure-Peaks aus eịnem Urinchromatogramm (1c).

Das Absorptionsmaximum (= Remissionsminimum) liegt in allen Fällen bei $280 \mathrm{~nm}$. Auffällig ist die nur sehr geringe Bandenverbreiterung in den Remissionsspektren im Vergleich zum Transmissionsspektrum (27). Der abgeflachte Verlauf der Remissionsspektren unterhalb von $240 \mathrm{~nm}$ dürfte auf den zunehmenden Anteil an regulärer Reflexion zurückzuführen sein (23).

Nachweisgrenze

Wie schon oben erwähnt, endet der lineare Bereich der Eichkurve bei $0,5 \mu \mathrm{g}$ Homovanillinsäure. Deswegen erscheint eine Messung unterhalb dieser Menge nicht sinnvoll, obwohl bei der gewählten Geräteeinstellung noch etwa $0,2 \mu \mathrm{g}$ Homovanillinsäure nachgewiesen werden können. $0,5 \mu \mathrm{g}$ Homovanillinsäure pro Fleck entsprechen bei der hier beschriebenen Arbeitsweise $1 \mathrm{mg}$ Homovanillinsäure/1 Urin.

\section{Ergebnisse und Diskussion}

Untersuchungen zum Verfahrien

Extraktion der Homovanillinsäure aus dem Urin

Zur Extraktion der Phenolcarbonsäuren aus dem Urin sind meistens Essigsäureäthylester oder Dichlormethan 


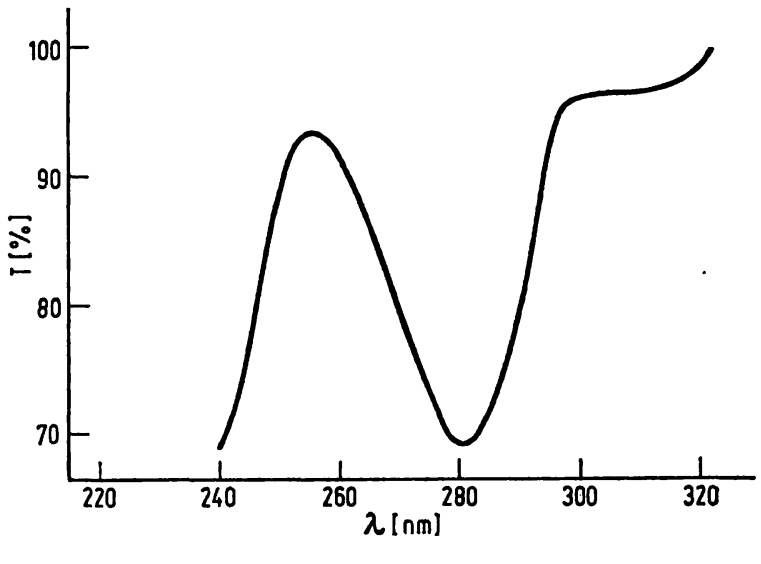

Abb. 1 a

Absorptionsspektrum von Homovanillinsäure in Äthanol

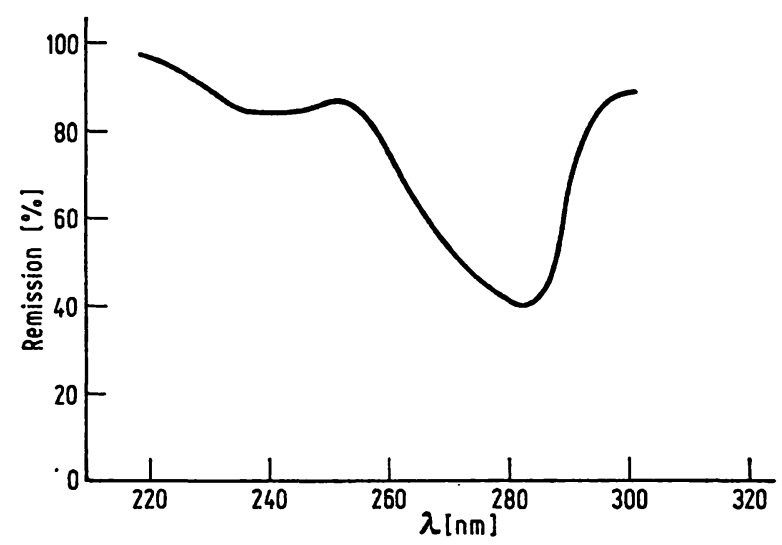

Abb. 1 b

Remissionsspektrum von reiner Homovanillinsäure auf Kieselgel $H$

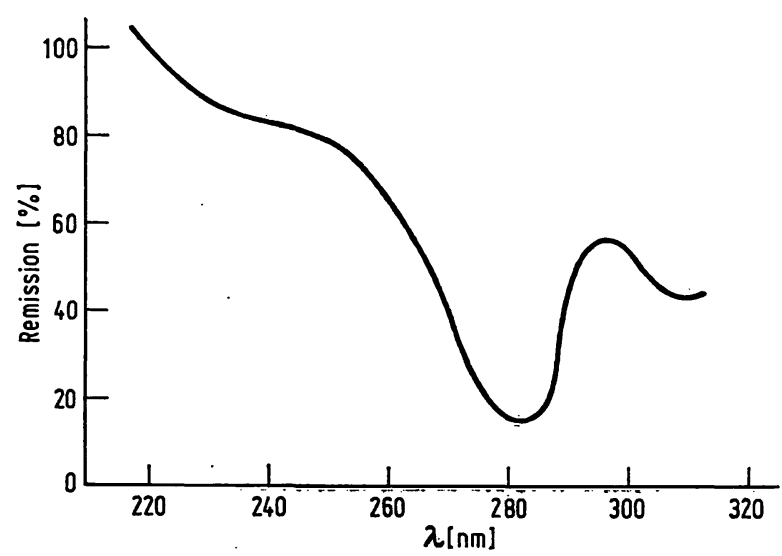

Abb. $1 \mathrm{c}$

Remissionsspektrum von Homovanillinsäure aus einem Urinextrakt

verwandt worden. Die Homovanillinsäure wird von beiden Lösungsmitteln gleich gut extrahiert, doch soll das Dichlormethan weniger Störsubstanzen mitschleppen (6). Wir haben Essigsäureäthylester als Extraktionsmittel gewählt, da sich in diesem Fall die spezifisch schwerere wäßrige Phase besser aus den spitz ausgezogenen Zentrifugengläsern entfernen läßt.

Es hat sich gezeigt, daß zur Extraktion der Homovanillinsäure der Zusatz von $\mathrm{NaCl}$ zum Urin nicht nur unnötig ist, sondern auch den weiteren Analysenverlauf stören würde. Wir haben deshalb auf die an dieser Stelle allgemein übliche Sättigung mit $\mathrm{NaCl}$ verzichtet.

\section{Variation der Schicbtdicke}

Als Schichtdicke des Adsorbens bei der dünnschichtchromatographischen Trennung der Phenolcarbonsäuren haben wir $250 \mu \mathrm{m}$ gewählt. Größere Schichtdicken erlauben zwar ein schnelleres Auftragen von größeren Substanzmengen, aber die Trennung wird schlechter. Bei geringeren Schichtdicken steigt zwar die Empfindlichkeit der Remissionsmessung bei besserer Auftrennung, jedoch wird beim Auftragen der Lösungen die Schicht leicht zerstört. Durch den starken Anstieg der Empfindlichkeit der Remissionsmessung unterhalb von $250 \mu \mathrm{m}$ Schichtdicke führen Schichtdickendifferenzen innerhalb einer Platte außerdem zu größeren Fehlern als bei dickeren Schichten (28).

\section{Wabl des Lösungsmittels zum Auftragen auf die Platte}

Ein schnelles Auftragen der Substanz ist um so einfacher, je tiefer der Siedepunkt des Lösungsmittels liegt. Ein zu leicht verdampfendes Lösungsmittel ist für eine exakte Volumendosierung ungünstig. $\mathrm{Zu}-$ sätzlich zeigten sich beträchtliche Empfindlichkeitsunterschiede bei der Remissionsmessung der Homovanillinsäure in Abhängigkeit von dem beim Auftragen verwendeten Lösungsmittel. Dieser Effekt ist dadurch bedingt, daß infolge der unterschiedlichen Löslichkeit der Homovanillinsäure in den einzelnen Lösungsmitteln und deren unterschiedlicher Polarität es zu einer verschiedenartigen Verteilung der Homovanillinsäure auf der Oberfläche des Sorptionsmittels kommt (28).

Die größte Empfindlichkeit bei der Remissionsmessung der Homovanillinsäure erzielt man durch Auftragen in Äthanol/Wasser $=3 / 2$. $\mathrm{Da}$ es mit diesem Lösungsmittelgemisch auch gelingt, die Homovanillinsäure quantitativ aus den Zentrifugengläsern nach dem Eindampfen des Essigsäureäthylesters wieder aufzunehmen, haben wir es zum Auftragen der Urinextrakte und Standards benutzt. Infolge des hohen Wasseranteils ist allerdings nur ein relativ langsames Auftragen möglich.

\section{Stabilität der Homovanillinsäure auf der Platte}

Kurve $I$ in Abbildung 2 zeigt die zeitliche Abhängigkeit der Peakfläche von $3 \mu \mathrm{g}$ Homovanillinsäure, wobei die Dünnschichtplatte geschützt vor direkter Sonneneinstrahlung aufbewahrt wird. Es ergibt sich ein kontinuierlicher Anstieg der Fläche, so daß nach 21,5 Stunden $140 \%$ des Ausgangswertes erreicht sind. Dieser Effekt läßt sich erklären als Folge einer Oxydation des Homovanillinsäure-Moleküls, wobei sich ein Produkt mit einem größeren Absorptionskoeffizienten bilden muß.

Diese Annahme läßt sich durch zwei Experimente stützen:

1. Eluiert man nach 3 Tagen den „Homovanillinsäure"-Fleck und führt eine erneute Chromatographie durch (Laufmittel nach (8)), so läßt sich gar keine Homovanillinsäure mehr nachweisen. 


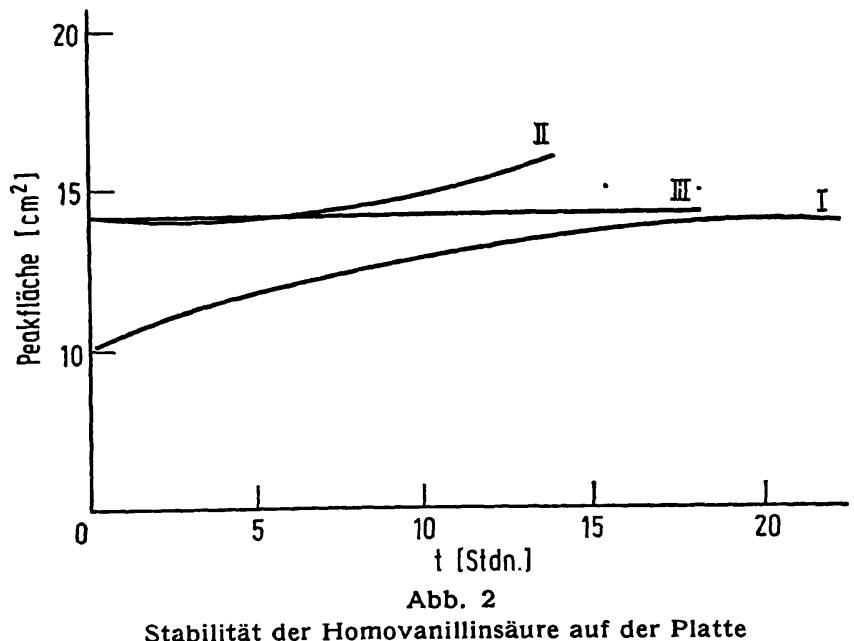

Kurve I: $3 \mu \mathrm{g}$ Homova Kurve II: $3 \mu \mathrm{g}$ Homovanillinsäure, $100 \mu \mathrm{l}$ 2-Mercapto-äthanol/100ml Laufmittel

Chromatographietank aufbewahrt, der mit 2-Mercapto-äthanol-Dämpfen gesättigt ist

2. Durch ein Antioxydationsmittel läßt sich die Oxydation der Homovanillinsäure verhindern. Durch Zusatz von 2-Mercapto-äthanol zum Laufmittel (100 $\mu 1 /$ $100 \mathrm{ml}$ Laufmittel) bleibt die Peakfläche 6 Stunden konstant (Kurve II in Abbildung 2). Nach dieser Zeit ist das 2-Mercapto-äthanol verdunstet.

Bewahrt man schließlich die Platte ohne vorhergehenden Oxydationsschutz im Laufmittel in einem Chromatographietank auf, in dem sich ein Schälchen mit 2Mercapto-äthanol befindet, so ist die Peakfläche nach 17,5 Stunden noch gleich dem Ausgangswert (Kurve III in Abbildung 2). Letzteres Verfahren empfiehlt sich, falls man die Platte vor der Messung längere Zeit (z. B. über Nacht) liegen lasșen muß.

\section{Dünnschicbtchromatographische Trennung der Phenolcarbon- säuren}

Als Laufmittel bei der dünnschichtchromatographischen Trennung der Phenolcarbonsäuren verwenden wir die Oberphase eines Gemisches Benzol/Eisessig/Wasser = 2/2/1 (v/v). Dieses Laufmittel ermöglicht eine gute Trennung der Homovanillinsäure von den anderen Phenolcarbonsäuren und hat den Vorteil einer großen Laufgeschwindigkeit $(15 \mathrm{~cm} / 35 \mathrm{Min}$.). Die Abbildungen $3 a-c$ zeigen die Verbesserung der Auftrennung durch Mehrfachchromatographie.

SANKOFF und SOURKES (6) verwenden die organische Phase eines Gemisches Benzol/Eisessig/Wasser $=2 / 3 / 1$ $(v / v)$ und Kieselgel $G$ als Adsorbens. Doch gelingt es ihnen nicht, die Homovanillinsäure von der Vanillinsäure zu trennen. Da sie nach Elution eine unspezifische Farbreaktion anschließen, müssen sie zu hohe Homovanillinsäure-Werte erhalten, da die Vanillinsäureausscheidung bei pflanzenreicher Ernährung $4-20 \mathrm{mg} /$ 24 Stdn. beträgt (29). Bei der in dieser Arbeit beschriebenen Methode hat die Vanillinsäure eine Laufstrecke von $10,5 \mathrm{~cm}$ und stört die Bestimmung der Homovanillinsäure (Laufstrecke $8,3 \mathrm{~cm}$ ) nicht.

Die bisher im Urin nachgewiesenen $(1,6,30)$ und mit Essigsäureäthylester bei $\mathrm{pH} 1-2$ extrahierbaren $\mathrm{Phe}$ nolcarbonsäuren haben wir in dem von uns benutzten chromatographischen System auf eine eventuelle Störung der Homovanillinsäure-Bestimmung geprüft. Das Ergebnis ist in Tabelle 3 zusammengefaßt.
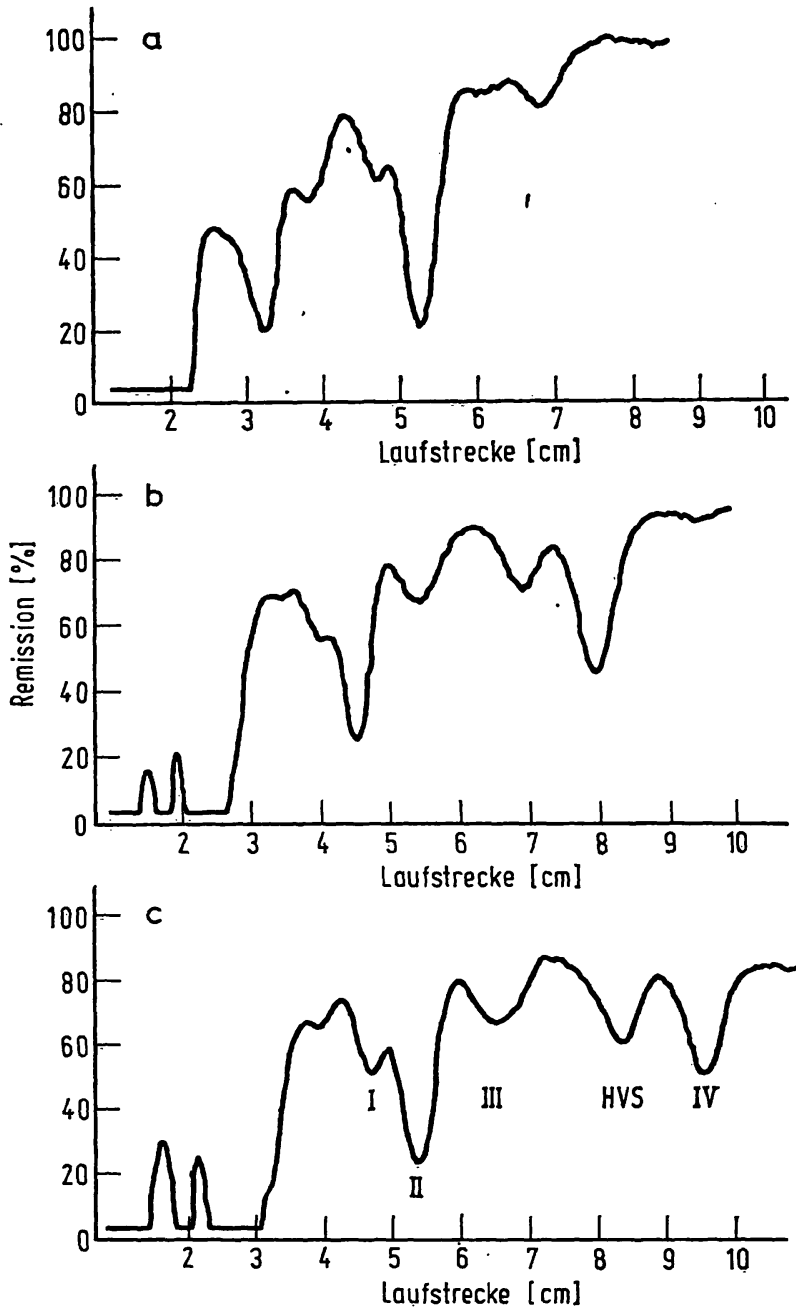

Abb. $3 a-c$

Chromatogramme eines Urinextraktes nach der 1., 2. und 3. Chromatographie. II = 3-Hydroxyphenylessigsäure, II = 4-Hydroxyphematographie. I = 3-Hydroxyphenylessigsäure, II = 4-Hydroxyphenylessigsäure, III = 3-Hydroxybenzoesäure, IV = nicht identifiziert,
HVS $=3$-Methoxy-4-hydroxy-phenylessigsäure (Homovanillinsäure)

Tab. 3

Laufstrecken der im Urin nachgewiesenen und mit Essigsäureäthylester bei pH $1-2$ extrahierbaren Phenolcarbonsäuren

\begin{tabular}{|c|c|}
\hline Phenolcarbonsäure & $\begin{array}{c}\text { Laufstrecke } \\
\text { (cm) }\end{array}$ \\
\hline 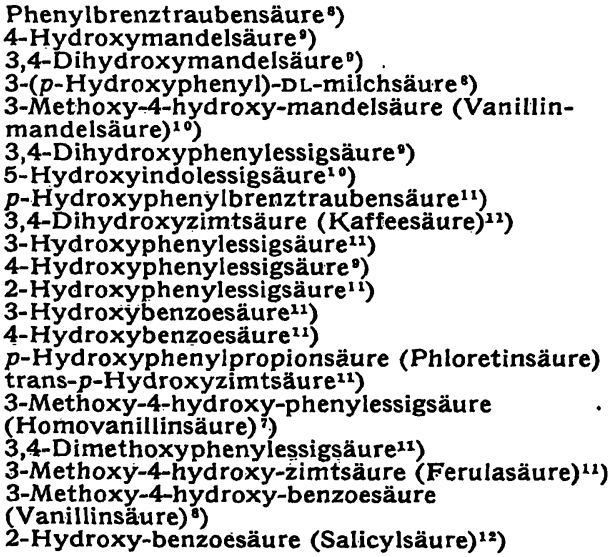 & $\begin{array}{r}0,2 \\
0,4 \\
0,4 \\
0,6 \\
1,2 \\
1,5 \\
2,3 \\
2,7 \\
3,2 \\
4,8 \\
5,3 \\
6,4 \\
6,5 \\
6,5 \\
6,8 \\
7,0 \\
8,3 \\
9,8 \\
10,2 \\
10,5 \\
12,2\end{array}$ \\
\hline
\end{tabular}

7) Fluka AG, Buchs/Schweiz o) Dr. Theodor Schuchardt, Mün1i) EGA-Chemie, Steinheim/Albuch 
Aus dieser Aufstellung geht hervor, daß keine der bisher im Urin nachgewiesenen Phenolcarbonsäuren die hier beschriebene Homovanillinsäurebestimmung stören kann. Abbildung 4 zeigt, daß die beiden Säuren, die trans-p-Hydroxyzimtsäure und die 3,4-Dimethoxyphenylessigsäure, die dem Homovanillinsäure-Peak benachbart sind, von diesem vollständig abgetrennt sind.

Dies bestätigt das Ergebnis, das durch Rechromatographie des „Homovanillinsäure-Peaks“ eines Urinextraktes in einem 2. Laufmittelsystem erhalten wurde.

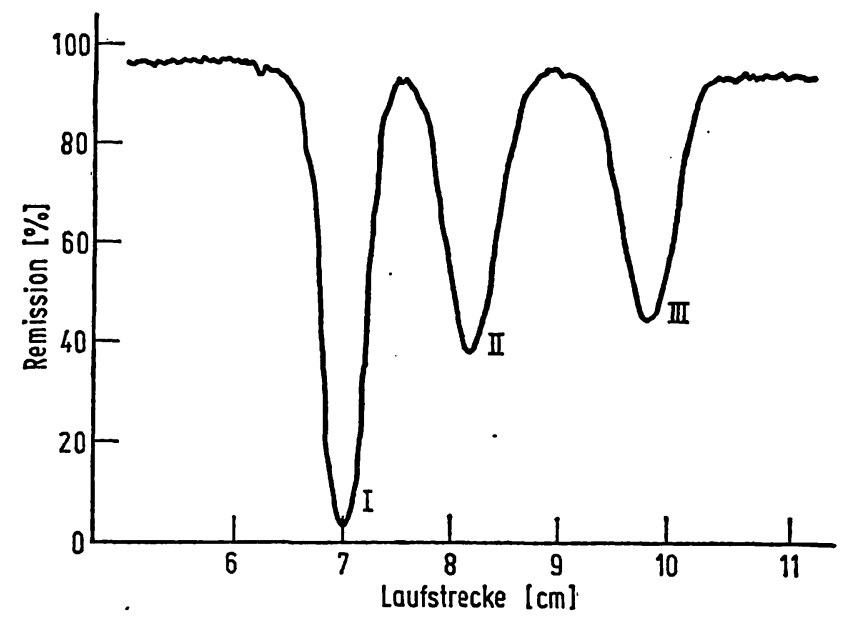

Abb. 4

Chromatogramm von trans-p-Hydroxyzimtsäure (I), Homovanillinsäure (II) und 3,4-Dimethoxyphenylessigsäure (III)

\section{Identifizierung weiterer Peaks}

Anhand der Laufstrecken der reinen Referenzsubstanzen sowie durch Aufstockungsversuche konnten wir die Identität der Peaks I, II und III (Abbildung 3c) aufklären.

Peak I : 3-Hydroxyphenylessigsäure

Peak II : 4-Hydroxyphenylessigsäure

Peak III: 3-Hydroxybenzoesäure.

Die 24-Stunden-Ausscheidung dieser Substanzen liegt im Milligramm-Bereich (30), was in guter Übereinstimmung steht $\mathrm{zu}$ den von uns ermittelten Peakflächen im Urinchromatogramm. Peak III ist nicht einheitlich, wahrscheinlich ist darin auch die 4Hydroxybenzoesäure enthalten.

Peak IV konnte noch nicht identifiziert werden. Zwar hat die 3,4-Dimethoxyphenylessigșäure die gleiche Laufstrecke, doch kommt diese Säure aufgrund ihrer geringen Ausscheidung (25-500 $\mu \mathrm{g} / 24$ Stdn. (31)) hierfür nicht in Frage. Durch Rechromatographie in dem Laufmittelsystem nach TAUTZ (7) korinten wir keine 3,4-Dimethoxyphenylessigsäure als Bestandteil des Peaks IV nachweisen.

\section{Anwendung der Methode}

\section{Analyse von 3-Stunden-Urinproben}

Die beschriebene Methode wurde angewendet zur Analyse von 3-Stunden-Urinproben bei 5 verschiedenen Versuchspersonen über jeweils 2 Tage. Nachts zwischen
23 und $8 \mathrm{Uhr}$ betrug die Sammelperiode 9 Stunden. In Abbildung 5 sind die Ergebnisse dieser Versuchsserie als prozentuale Abweichung vom Mittelwert dargestellt. Es ist daraus zu ersehen, daß die Ausscheidung der Homovanillinsäure tageszeitabhängig ist, wobei das Minimum der Ausscheidung zwischen 23 und $8 \mathrm{Uhr}$ liegt. Auffallend sind die geringen Amplituden.

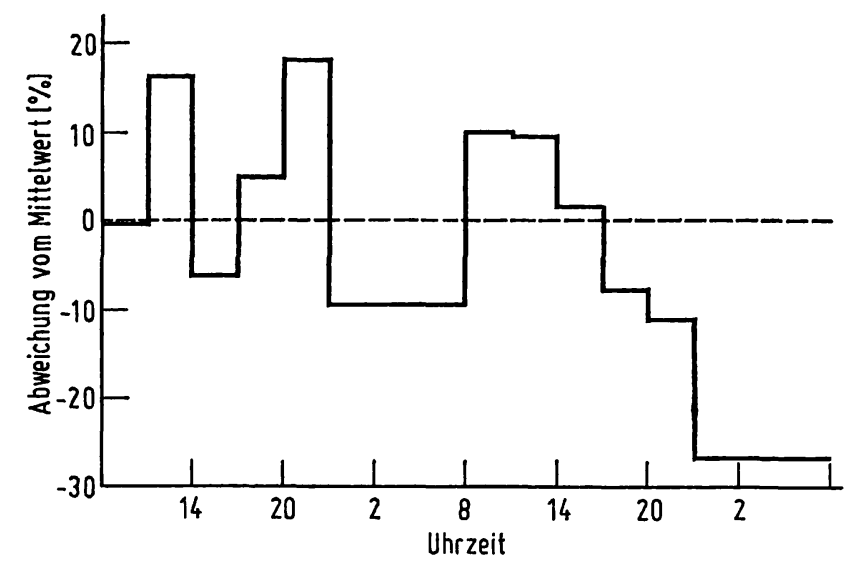

Abb. 5

Tageszeitliche Schwankungen der Homovanillinsäure-Ausscheidung bei 5 Versuchspersonen als proz. Abweichung vom Mittelwert über 2 Tage

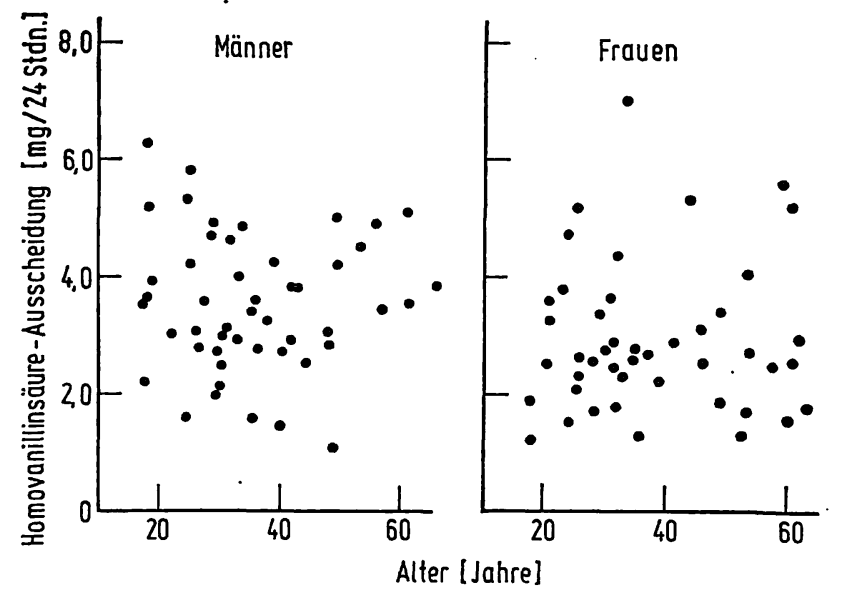

Abb. 6

Homovanillinsäure-Ausscheidung im 24-Stunden-Urin bei 45 Frauen und 49 Männern in Abhängigkeit vom Alter

\section{Normalwerte}

Die Homovanillinsäure-Ausscheidung in 24-StundenUrinen wurde an einem Kollektiv von 45 Frauen und 49 Männern im Alter zwischen 18 und 65 Jahren, die ihrer normalen Beschäftigung nachgingen, bestimmt.

Die Ergebnisse sind in Abbildung $6 a$ und $b$ für Männer und Frauen getrennt aufgezeichnet. Wie aus dieser Abbildung hervorgeht, besteht keine Altersabhängigkeit der Homovanillinsäure-Ausscheidung.

Wie bei der 24-Stunden-Ausscheidung von Adrenalin, Noradrenalin, Dopamin und Vanillinmandelsäure ist auch bei der Homovanillinsäure der Median bei den Frauen tiefer als bei den Männern. Dieser Unterschied ist statistisch signifikant (WILcoxoN-Test, $P<0,01$ ). Dies wurde bei der gleichen Stichprobe auch schon für Adrenalin und Dopamin gefunden, während für die 
Tab. 4

Normalbereich der Homovanillinsäure-Ausscheidung in $\mathrm{mg} / 24 \mathrm{Stdn}$.

\begin{tabular}{ccc}
\hline Kollektiv & Median & 96 Percentil \\
\hline Männer $\mathrm{n}=49$ & 3,56 & $1,44-6,25$ \\
Frauen $\mathrm{n}=45$ & 2,60 & $1,27-5,57$ \\
Gesamt $\mathrm{n}=94$ & 3,02 & $1,27-6,25$ \\
\hline
\end{tabular}

Noradrenalin- und Vanillinmandelsäureausscheidung im 24-Stunden-Urin kein signifikanter Geschlechtsunterschied bestand (32).

In Tabelle 5 sind zum Vergleich die in der Literatur angegebenen Normalwerte, Größe der Kollektive, benutzte Methode sowie Literaturzitat zusammengefaßt. Der von uns ermittelte Normalbereich stimmt gut mit dem von PETrÁšEK und Mitarbeitern (20) überein, doch ist deren Mittelwert höher als der Median unserer Stichproben. Bei allen anderen Methoden - mit Ausnahme von (33) - liegt der Normalbereich höher als bei dem hier beschriebenen Verfahren, doch sind die Kollektive meist zu klein.

Wir führen unseren niedrigeren Normalbereich auf die größere Spezifität dieser Methode zurück, insbesondere im Vergleich zu SANkoff und Sourkes (6), was an anderer Stelle, schon näher erläutert wurde.

Zur Gewinnung des Normalbereiches der Homovanillinsäure-Ausscheidung wurden die gleichen Urinproben benutzt, aus denen bereits die Werte für die 24-Stunden-Dopaminausscheidungen bestimmt worden waren. Es wurde geprüft, ob eine Korrelation der Dopamin- mit der Homovanillinsäure-Ausscheidung besteht. Die Produkt-Moment-Korrelationskoeffizienten betrugen bei den Männern 0,06 und bei den Frauen 0,48 . Auffällig ist dabei der Geschlechtsunterschied, für den keine eindeutige Erklärung gegeben werden kann. Nach diesen Werten besteht jedoch keine eindeutige Korrelation dieser Größen.

Der diagnostische Wert der Homovanillinsäure-Bestimmung

Tumoren, die sich vom sympathischen Gewebe (Neuroblastom, Ganglioneuroblastom, Ganglioneurom) oder chromaffinen Gewebe (Phäochromoblastom, Phäochromocytom) ableiten, führen zu einer erhöhten Ausscheidung der Katecholamine und deren Abbauprodukten im Harn (34).

Histologisch, klinisch und biochemisch bestehen Unterschiede zwischen den beiden Tumorarten, ebenso in der Ausscheidung der Katecholamine und ihrer Metabolite im 24-Stunden-Urin. Beim Phäochromocytom werden Adrenalin oder Noradrenalin, die Metanephrine und die Vanillinmandelsäure vermehrt ausgeschieden. Kommt es zu maligner Entartung, werden außerdem Dopa, Dopamin und Homovanillinsäure vermehrt ausgeschieden. Tumoren, die sich von den Sympathoblasten oder Sympathocyten ableiten, scheiden neben Dopa, Dopamin und Noradrenalin ihre 3-Omethylierten Derivate vermehrt im Urin aus, d. $h$. 3-Methoxytyramin, Homovanillinsäure, Normetanephrin und Vanillinmandelsäure. Das Ausscheidungsmuster des sehr seltenen Phäochromoblastoms ist dem. des Neuroblastoms ähnlich. Mit Hilfe der Homovanillinsäure ist also im Einzelfall keine eindeutige differentialdiagnostische Entscheidung, ob ein Tumor des chromaffinen oder sympathischen Gewebes vorliegt, möglich. Für die Diagnostik eines Neuroblastoms ist die relativ einfache und zuverlässige Bestimmung der Vanillinmandelsäure der der Homovanilliñsäure überlegen oder zumindest gleichwertig, wie aus den in der folgenden Tabelle zusammengestellten Literaturangaben hervorgeht.

Tab. 6

Die Ausscheidung der Homovanillinsäure (HVS) und Vanillinmandelsäure (VMS) bei Tumoren der Neuralleistè. Die erste Ziffer gibt die Zahl mit erhöhter Ausscheidung und die zweite die Gesamțahl an

\begin{tabular}{|c|c|c|c|}
\hline $\begin{array}{c}\text { Literaturzitat } \\
\text { Nr. }\end{array}$ & $\begin{array}{c}\text { VMS } \\
\text { Ausscheidung }\end{array}$ & $\begin{array}{c}\text { HVS } \\
\text { Ausscheidung }\end{array}$ & Tumor \\
\hline 9,34 & $24 / 25$ & $17 / 25$ & Neuroblastom \\
\hline \multirow[t]{2}{*}{35} & $\begin{array}{c}69 / 73 \\
3 / 6\end{array}$ & $\begin{array}{c}24 / 36 \\
2 / 4\end{array}$ & $\begin{array}{l}\text { Neuroblastom } \\
\text { Ganglioneuro- } \\
\text { blastom }\end{array}$ \\
\hline & $2 / 8$ & $1 / 6$ & Ganglioneurom \\
\hline 20 & $17 / 18$ & $18 / 18$ & Neuroblastom \\
\hline
\end{tabular}

Tab. 5

Literaturübersicht

Die Homovanillinsäure-Ausscheidung in 24-Stunden-Urinen mit verschiedenen Methoden bestimmt $\mathrm{PC}=$ Papierchromatographie; DC = Dünnschichtčhromatógraphie

\begin{tabular}{|c|c|c|c|c|c|}
\hline $\begin{array}{l}\text { Normalbereich } \\
\text { mg/24 Stdn. }\end{array}$ & $\mathrm{mg} / 24$ Stdn. & $\mathbf{n}$ & Besonderheiten & Methode & Literatur \\
\hline $3-8$ & & & Einige hundert gesunde Erwachsene & zweidimensionale PC & $\begin{array}{c}2 \\
(1957)\end{array}$ \\
\hline $4,5-15,6$ & 8,23 & & & $\begin{array}{l}\text { DC + Elution des mit Folins Phenol- } \\
\text { Reagenz geb. Farbstoff }\end{array}$ & $\begin{array}{c}6 \\
(1963)\end{array}$ \\
\hline $3,4-7,3$ & 5,1 & 18 & gesunde Erwachsene & Demethylierung im Autoklaven, Farbreaktion & $\begin{array}{c}17 \\
(1964)\end{array}$ \\
\hline $3,7-7,5$ & 5,4 & 5 & gesunde Erwachsene & Fluorometrie & $\begin{array}{l}16 \\
(1965)\end{array}$ \\
\hline $4,2-7,7$ & 5,35 & 15 & gesunde Erwachsene & $\begin{array}{l}\text { Oberführung in Vanillin im Autoklaven, } \\
\text { spektrophotometrische Bestimmung }\end{array}$ & $\begin{array}{l}18 \\
(1966)\end{array}$ \\
\hline $1,5-7,5$ & 4,65 & & gesunde Frauen und Männer & (a) & $\begin{array}{l}20 \\
(1966)\end{array}$ \\
\hline & & & $\cdot+$ & $\begin{array}{l}\text { zweidimensionale PC und Farbreaktion, } \\
\text { Elution }\end{array}$ & $\begin{array}{c}4 \\
(1966)\end{array}$ \\
\hline $1,1-3,8$ & 2,46 & 8 & melanomfreie Kontrollpersonen & zweidimensionale PC & $\begin{array}{c}33 \\
(1966)\end{array}$ \\
\hline
\end{tabular}




\section{Diragrą)

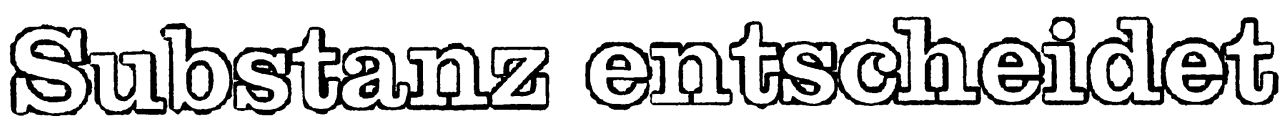

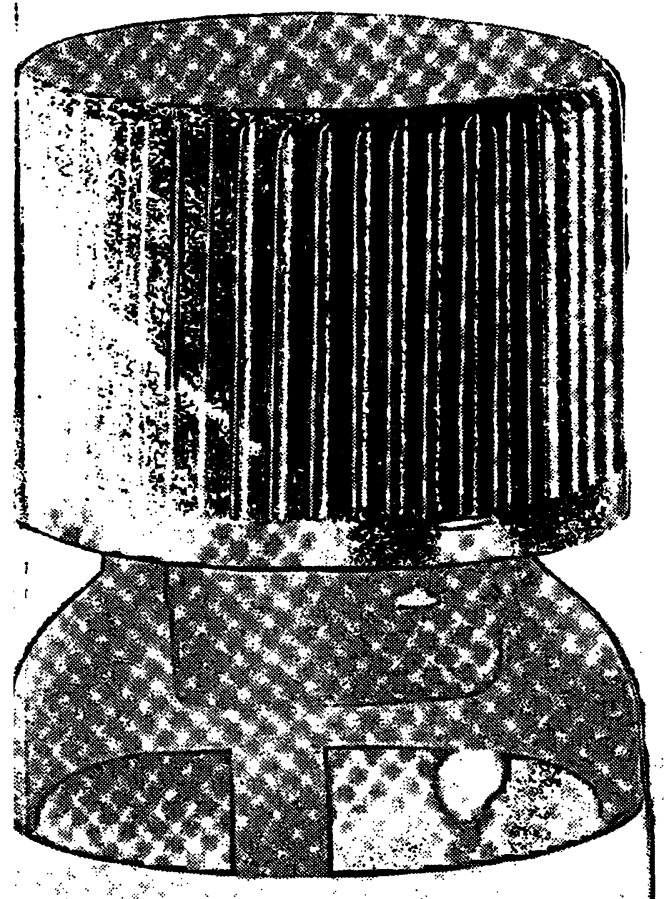

Merck-1-Test GOT (UV)

(3)

Differentislagnose vou Herz- und Lebererkrankangen

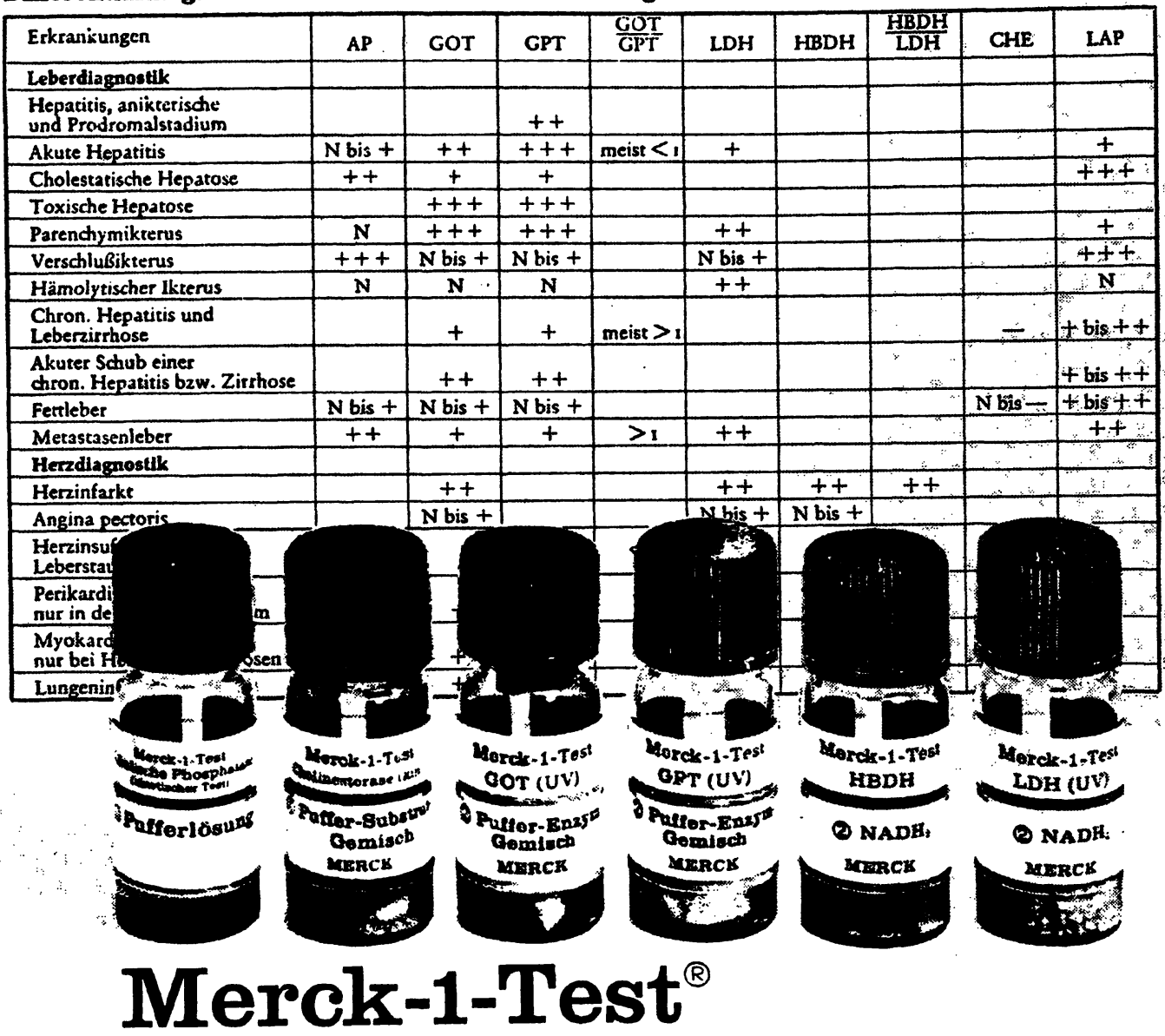

Das moderne Konzept für Sicherheit und Zeitersparnis in Klinik- und Praxislabor bietet ein vollständiges Grundsortiment wichtiger Enzymtests für die Herzund Leberdiagnostik.

Merck-1-Test ${ }^{(1)}$ Alkalische Phosphatase (kin. Test)

Merck-1-Test Cholinesterase (kin. Test)

Merck-1-Test ${ }^{\boxplus}$ GOT (UV)

Merck-1-Test ${ }^{(i)}$ GPT (UV)

Merck-1-Test ${ }^{\text {i, }}$ HBDH (UV)

Merck-1-Test LDH (UV)

Merck-1-Test Leucin-Arylamidase, sog. LAP (kin. Test)

Merck-1-Test ${ }^{\text {in }}$ Hämoglobin

Verlangen Sie bitte unseren Spezial-Prospekt.

E. Merck, Darmstadt 


\section{Niedermolekulare Substanzen schnell und wirksam trennen}

mit der Hochspannungs-

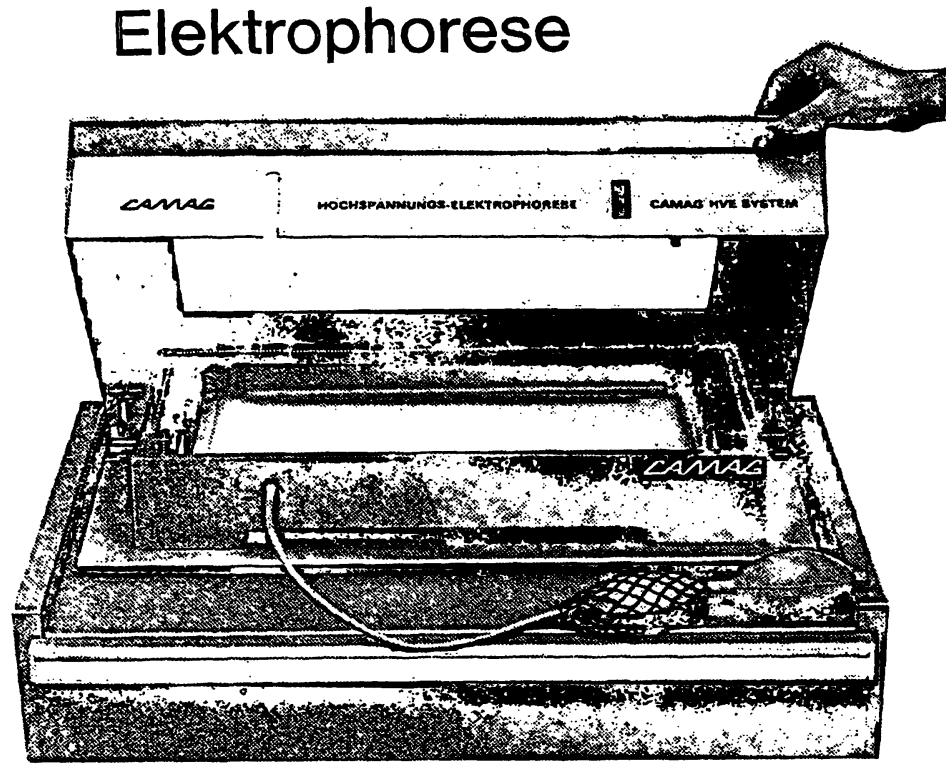

\section{Mit dem 124 -System} dauert z.B. eine

Aminosäure-Trennung knapp 15 Minuten

\section{Weitere Beispiele: Oligopeptide \\ Kohlenhydráte \\ Porphyrine \\ Phenole \\ Vitamine}

Das CAMAG-System ist besonders wirtschaftlich, sicher und unkompliziert.

Verlangen Sie unseren Prospekt - oder noch besser eine

\section{Demonstration in Ihrem Labor}

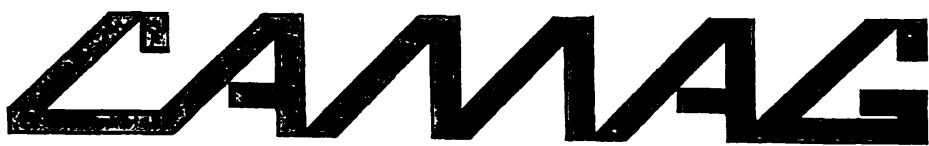

Chemie-Erzeugnisse und Adsorptionstechnik AG
4132 Muttenz/Schweiz

Homburgerstrasse 24

Telefon (061) 531430
1000 Berlin 45

Baseler Strasse 65

Telefon (0311) 737077

\section{W Walter de Gruyter Berlin·NewYork}

\section{Biltz-Klemm-Fischer \\ Experimentelle Einführung in die anorganische Chemie}

Neu herausgegeben von W. Klemm und W. Fischer. 63.-70. Auflage. Oktav. XII, 228 Seiten. 1970. Balacron flexibel DM 21,-

Die Kürzung der Dauer des Chemiestudiums führt besonders zu einer Kürzung der experimentellen Ausbildung, andererseits müssen im Anfang grundlegende Stoffkenntnisse anhand der wichtigsten Ordnungsprinzipien vermittelt werden.

Um den heutigen Forderungen einer solchen Einführung Rechnung zu tragen, wurde das Werk überarbeitet und teilweise geändert.

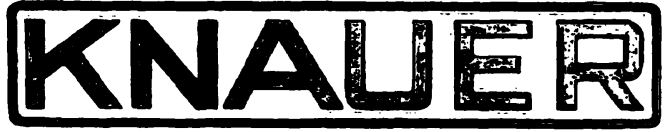

ELEKTRONISCHES HALBMIKRO-OSMOMETER

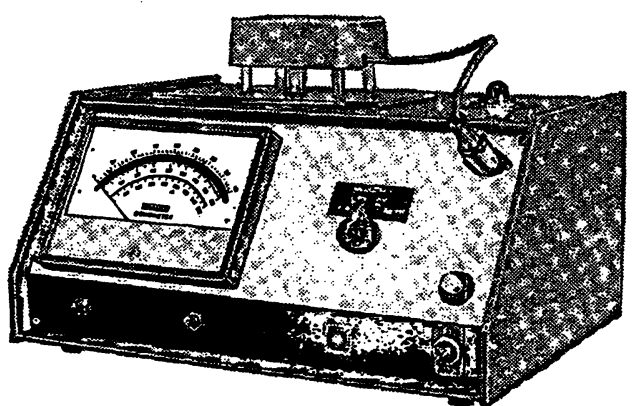

zur direkten Bestimmung der Osmolalität aller Körperflüssigkeiten wie Blut, Serum, Urin, Liquor durch Gefrierpunktmessung.

Kontrolle der Nierenfunktion

Kontrolle von iso-, hyper- und hypotonischen Lösungen Prüfung von Infusionslösungen

- Probevolumen nur $0,15 \mathrm{ml}$ oder $0,05 \mathrm{ml}$

- Betriebsbereitschaft sofort nach dem Einschalten

- Daver einer Messung ca. 2 Minuten

- Meßgenauigkeit 1-2 Milliosmol/kg bzw. 1\%

- Preis DM 3600,- + MWSt

- Lieferung ab Lager oder laufenden Serien

Weitere Spezialität: Komplettes System zur Molekulargewichtsbestimmung zwischen 100 und 1000000 durch Kryoskopie, Dampidruck-Osmometrie und Membran-Osmometrie.

Wissenschaftlicher Gerätebau

KG Dr.-Ing. Herbert Knaver \& Co. GmbH,

1 Berlin 37 (West), Holstweg 18, Tel. (0311) 848705 
Im Gegensatz zu KüSER (35) fand PetrášEK (20) in allen Fällen mit Neuroblastom eine erhöhte Homovanillinsäure-Ausscheidung. Nach der Untersuchung von KÄSER nimmt mit zunehmender Reife des Gewebes die Anzahl der Fälle mit normaler Ausscheidung sowohl von Vanillinmandelsäure wie von Homovanillinsäure zu. Außerdem konnte KäsER zeigen, daß für die Diagnostik des Neuroblastoms die Bestimmung der Dopamin-Ausscheidung von besonderem diagnostischem Wert ist; denn von 34 Patienten mit einem
Neuroblastom zeigten nur 2 eine normale Ausscheidung.

Da Neuroblastome mit normaler Vanillinmandelsäure. bei erhöhter Homovanillinsäure-Ausscheidung beschrieben sind $(9,36)$, bedeutet die Bestimmung der letzteren Verbindung eine zusätzliche Sicherung. Neben ihrer Bedeutung im Rahmen der Diagnostik ist ihre Bestimmung von Nutzen zur Therapiekontrolle.

Fräulein Jutta-Maria QUANTE danken wir für ihre sorgfältige technische Mitarbeit.

\section{Literatur}

1. Armstrong, M. D., K. N. F. Shaw und P. E. Wall, J. biol. Chemistry 218, 293 (1956). - 2. SHAw, K. N. F., A. McMrLLAN und M. D. Armstrong, J. biol. Chemistry 226, 255 (1957). 3. Axelrod, J., S. Senoh und B. Wrtkop, J. biol. Chemistry 233, 697 (1958). - 4. Comoy, E., Ph. Brunelle und B. Parrad, Ann. Biol. clin. 24, 655 (1966). - 5. Duke, Pr. S. und H. B. Demopoulos, Clin. Chem. (New York) 14, 212 (1968). - 6. SankofF, I. und T. L. Sourkes, Canad. J. Biochem. 41, 1381 (1963). - 7. TAutz, N. A., G. Voltmer und E. Schmi, Klin. Wschr. 43, 233 (1965). - 8. Schmid, E., B. LAUdr, J. KraUtHEIM und N. A. TAUTZ, diese Z. 4, 250 (1966). - 9. StudnItz, W. v., Klin. Wschr. 40, 163 (1962). - 10. Wrlliams, C. M. und Ch. C. Sweeley, J. Clin. Endocr. (Springfield) 21, 1500 (1961). 11. Sweelex, CH. C. und C. M. Wrlliams, Analytic. Biochem. 2, 83 (1961). - 12. Williams, C. M., Analytic. Biochem. 4, 423 (1962). - 13. Williams, C. M. und M. Greer, Clin. chim. Acta (Amsterdam) 7, 880 (1962). - 14. Sharman, D. F., Brit. J. Pharmacol. 20, 204 (1963). - 15. ANdén, N.-E., B.-E. Roos und B. Werdintus, Life Sci. 2, 448 (1963). - 16. Sato, T. L., J. Laborat. Clin. Med. (S. Louis) 66, 517 (1965). - 17. Ruthven, C. R. J. und M. Sandler, Analytic. Biochem. 8, 282 (1964). - 18. Ruthven, C. R. J. und M. Sandler, Clin. chim. Acta (Amsterdam) 14, 511 (1966). - 19. Studnitz, W. v., persönliche Mitteilung. - 20. Petrišsek, J., J. Dubovský und J. Charvàt, Endokrinologie 50, 308 (1966). - 21. WäsSLE, W. und K. SANDHOFF, J. Chromatogr. 34, 357 (1968). - 22. Wisser, H. und D. Stamm, diese Z. 8, 21 (1970). - 23. Jork, H., Z. analyt. Chem. 221, 17 (1966). - 24. STAHL, E. und H. JorK, Zeiss Informationen 16 , 52 (1968). - 25. Patakr, G., Chromatographia 1, 492 (1968). 26. Zerss, C., Oberkochen/Württ., Druckschrift G 50-657/K/ II-d. - 27. JoRk, H., Internat. Chromatogr. Symp., Brüssel 1966, S. 227. - 28. JoRk, H., J. Chromatogr. 48, 372 (1970). 29. Dirscherl, W., A. Wirtzfeld, B. Brise und H. Thomas, Angew. Chem. 75, 140 (1963). - 30. Tompsetr, S. L., Clin. chim. Acta (Amsterdam) 3, 149 (1958). - 31. FriedhofF, A. J. und K. Furrya, Nature (London) 214, 1127 (1967). - 32. WisSER, H., diese Z. 8, 637 (1970). - 33. Schwartze, G. und G. Conradi, Der Hautarzt 17, 348 (1966). - 34. GJessing, L. R., Adv. Clin. Chem. 11, 81 (1968). - 35. KäSER, H., Pharmacol. Rev. 18, 659 (1966). - 36. Studnitz, W. v., Pharmacol. Rev. 18, 645 (1966).

Dr. E. Knoll

PD Dr. Dr. D. Stamm

Max-Planck-Institut für Psychiatrie

8 München 23

Kraepelinstr. 10

Dr. Dr. H. Wisser

zur Zeit:

Institut für Klin. Chemie der

Medizinischen Hochschule Hannover 